\title{
Global analysis of transcriptional regulators in Staphylococcus aureus
}

Jose Antonio Ibarra ${ }^{1,3^{*}}$, Ernesto Pérez-Rueda ${ }^{2^{*}}$, Ronan K Carroll ${ }^{1}$ and Lindsey N Shaw ${ }^{1}$

\begin{abstract}
Background: Staphylococcus aureus is a widely distributed human pathogen capable of infecting almost every ecological niche of the host. As a result, it is responsible for causing many different diseases. S. aureus has a vast array of virulence determinants whose expression is modulated by an intricate regulatory network, where transcriptional factors (TFs) are the primary elements. In this work, using diverse sequence analysis, we evaluated the repertoire of TFs and sigma factors in the community-associated methicillin resistant S. aureus (CA-MRSA) strain USA300-FPR3757.

Results: A total of 135 TFs and sigma factors were identified and classified into 36 regulatory families. From these around $43 \%$ have been experimentally characterized to date, which demonstrates the significant work still at hand to unravel the regulatory network in place for this important pathogen. A comparison of the TF repertoire of $S$. aureus against 1209 sequenced bacterial genomes was carried out allowing us to identify a core set of orthologous TFs for the Staphylococacceae, and also allowing us to assign potential functions to previously uncharacterized TFs. Finally, the USA300 TFs were compared to those in eleven other $S$. aureus strains including: Newman, COL, JH1, JH9, MW2, Mu3, Mu50, N315, RF122, MRSA252 and MSSA476. We identify conserved TFs among these strains and suggest possible regulatory interactions.
\end{abstract}

Conclusions: The analysis presented herein highlights the complexity of regulatory networks in S. aureus strains, identifies key conserved TFs among the Staphylococacceae, and offers unique insights into several as yet uncharacterized TFs.

Keywords: Transcriptional regulators, Virulence, Gene evolution, Staphylococacceae, Firmicutes

\section{Background}

Staphylococcus aureus is a facultative human pathogen and the casual agent of a diverse array of diseases, including superficial skin and wound-related tissue infections, food poisoning, bacteremia, endocarditis and pneumonia. This organism produces a diverse array of virulence factors, including toxins, adhesins, colonization and biofilm factors. S. aureus has obtained notoriety in recent years due to the appearance and worldwide spread of antibiotic resistant strains. Hospital associated (HA) and community associated (CA) infections caused by methicillin-resistant S. aureus (MRSA) have become a major public health

\footnotetext{
* Correspondence: ibarraj@usf.edu; erueda@ibt.unam.mx

'Department of Cell Biology, Microbiology and Molecular Biology, University of South Florida, 4202 East Fowler Avenue, ISA 2015, Tampa, FL 33620-5150, USA

${ }^{2}$ Departamento de Ingeniería Celular y Biocatálisis, Instituto de Biotecnologia UNAM, Av Universidad 2001, Cuernavaca, Morelos CP 62210, Mexico Full list of author information is available at the end of the article
}

concern, particularly for CA-MRSA infections as they cause life threatening disease in otherwise healthy individuals with no pre-existing risk factors [1]. Furthermore, CA-MRSA strains are replacing HA-MRSA strains in clinical settings, increasing the risk of transmission not only to patients but also into healthy individuals in the community (reviewed in [2]). As virulence determinant production is very tightly regulated in S. aureus, a thorough understanding of its regulatory network is necessary to fully comprehend the pathogenic processes of this bacterium. Additionally, exploring the regulatory differences between CA-MRSA and other MRSA strains may aid our understanding of the increase in virulence observed amongst community-associated isolates.

The relatively small size of Staphylococcal genomes, and their adaptability, suggests that these bacteria have a high degree of genome plasticity, depending on their environment $[3,4]$. Given the high number of virulence factors present in these bacteria, and the niche-specific

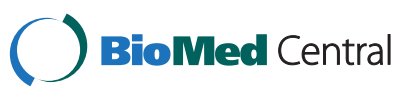


role many of them play during different stages of the infectious process, gene expression must be finely tuned in order to efficiently coordinate their expression, and also continue to preserve energy pools. In this context, DNAbinding transcription factors (TFs) play an important regulatory role by either repressing or activating genes in response to environmental and physiological conditions.

Even though diverse strains of $S$. aureus have been extensively studied, and subjected to genome sequencing, the function of a large proportion of their genes remains unidentified. In this work, we define the TF repertoire for the CA-MRSA strain USA300-FPR3757 and classify it into regulatory families. We have evaluated the orthologous distribution of these elements in other sequenced bacterial genomes using the repertoire of TFs identified in USA300, and identified a core set of regulators for both the Firmicutes phylum, and the Staphylococacceae group. Finally, we examine the conservation of 135 USA300 TFs amongst 11 other S. aureus strains, identifying a key group of regulators that display a high degree of conservation, including many that have previously been demonstrated to play a role in virulence gene regulation. We also highlight cases whereby TFs are absent, or altered within strains, suggesting changes in the wiring of regulatory networks in individual isolates.

\section{Results}

Identification of TFs and $\sigma$ factors in S. aureus USA300 In order to identify the repertoire of TFs in $S$. aureus we focused on the recently emerged CA-MRSA strain USA300-FPR3757. This strain was selected for a number of reasons: Firstly, USA300 is the most prevalent CA-MRSA strain, associated with outbreaks in the USA, Canada and Europe [5,6]. Secondly, USA300 strains exhibit fewer genomic changes amongst isolated strains than other MRSA lineages, suggesting they originate from a common clone [7]. Finally, USA300 strains display hyper-virulence using various animal models of infection [8]. Therefore, we performed an extensive search for possible TFs using database assignments, Hidden Markov model (HMMs) profiles, BLAST similarities and literature searches. From this, 135 TFs and $\sigma$ factors were identified in this strain. These putative regulators can be classified into 36 regulatory families (Figure 1 and 2), with only 2 classified as unknown. The largest TF family identified correspond to the MarR family (18 members), which includes the Sar subfamily; followed by the two component system response regulators (TCS-RR), with 16 members; followed by the GntR/DeoR family, and the Xre family (13 members each). Of interest, almost half of these elements have not been functionally characterized whatsoever (58 out of 135, 42.9\%). For this reason, and to begin to understand the role of these uncharacterized TFs in $S$. aureus, we sought to explore potential functions for them by identifying similarities in sequence and genomic context with well-known proteins identified in other bacterial species. A complete summary of these findings is presented in Table 1. Collectively, 34 of the 58 uncharacterized TFs could be assigned a putative function via bioinformatics analysis. Together these results show the apparent variety

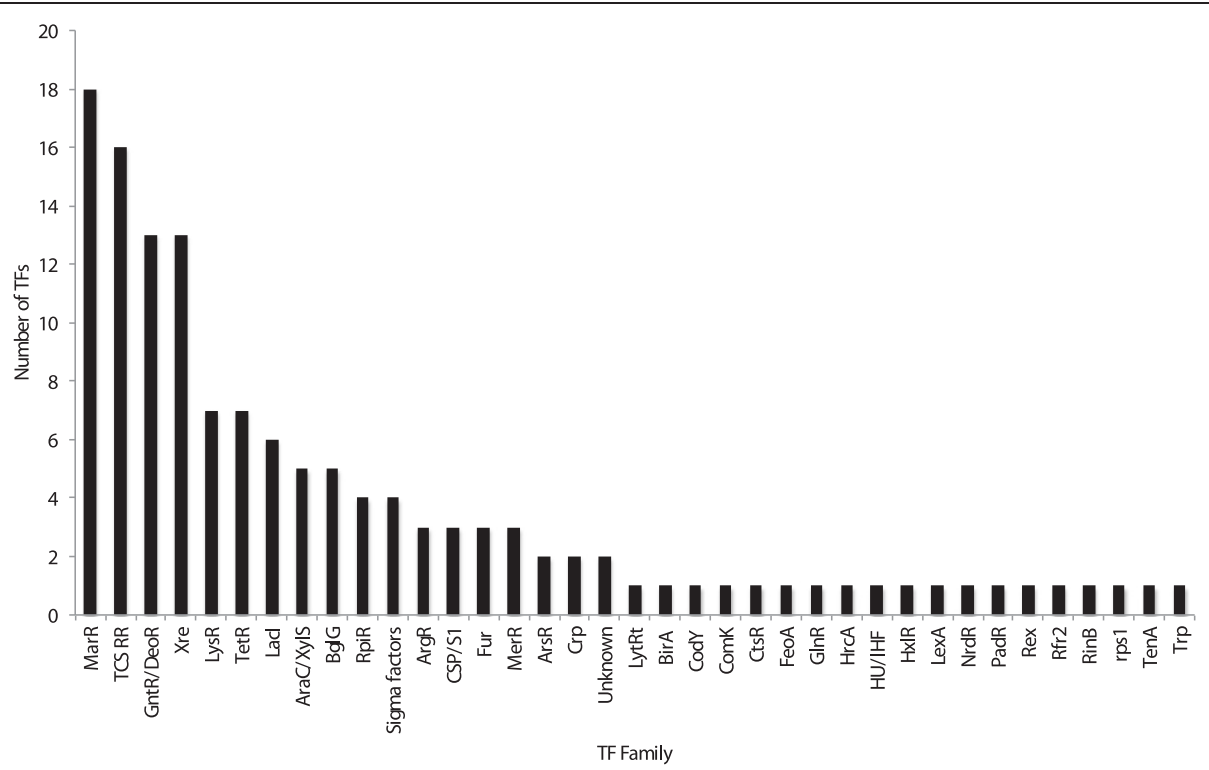

Figure 1 Classification of transcriptional factors (TFs) in S. aureus strain USA300-FPR3757. TFs were grouped after performing BLAST analyses and manual searches for several regulatory families. Those with no known family were placed in the "unknown" group. 
Figure 2 Comparison of USA300-FPR transcriptional factors (TFs) with other S. aureus strains. TFs identified in S. aureus USA300-FPR3757 were used to identify their orthologues in other S. aureus strains using BLAST and other tools (see Methods section). Presence of TFs is denoted by green and the absence by red colors; $\mathrm{T}$ indicates a truncated protein.

of TFs in S. aureus USA300, and indicate that a large majority of them are not well-characterized. This presents an obvious gap, and the need for additional research to explore the complex, diverse and understudied regulatory circuits of this important human pathogen.

\section{Distribution of USA300 TF homologs in eubacterial species}

Many bacterial TFs involved in key cellular processes are essential to the cell and are highly conserved. We hypothesized that a subset of the 135 TFs identified in USA300 would be conserved across eubacterial organisms. To test this hypothesis we set out to identify which TFs shared an orthologous protein in other bacterial phyla. A total of 1209 bacterial genomes were studied, comprising strains from the following phyla: Acidobacteria, Actinobacteria, Aquificae, Bacteroidetes, Chlamydiae, Chlorobi, Chloroflexi, Chrysiogenetes, Cyanobacteria, Deferribacteres, Deinococcus-Thermus, Dictyoglomi, division WWE1, Elusimicrobia, Fibrobacteres, Firmicutes, Fusobacteria, Gammatimonadetes, Nitrospirae, Planctomycetes, Proteobacteria, Spirochaetes, Synergistetes, Tenericutes, Thermobaculum, Thermatogae and Verrucomicrobia.

Based on a clustering analysis we classified TFs into 4 main groups (Figure 3): Group 1 included orthologues highly conserved across most of the phyla $(60-100 \%$ of organisms); Group 2, TFs less conserved in the diverse phyla analyzed (15-59\%); Group 3 included mostly Firmicutes specific TFs (1-14\%); and Group 4, those specific to Staphylococacceae $(<1 \%$ of genomes, but abundant in this group). Nine proteins were found in group 1 , suggesting an ancient origin for these regulators and perhaps playing a fundamental role in bacterial physiology (Additional file 1: Table S1). The one outstanding example of these is SAUSA300_1521 (sigma A, $\sigma^{\mathrm{A}}$ ), essential for housekeeping transcription in bacteria. Two proteins less conserved in this group are SAUSA300_1362 (HU) and SAUSA300_2480 (CidR), involved in genome packing, and the regulation of murein synthesis gene expression, respectively; although they are less conserved in Dictyoglomi, division WWE1 and Elusimicrobia. This suggests that organisms in those groups perhaps use alternative proteins to package their DNA. Group 2 includes several proteins widely distributed amongst all bacterial phyla, except in organisms with smaller genomes where gene loss 
Table 1 Possible role for uncharacterized TFs in S. aureus USA300

\begin{tabular}{|c|c|c|c|}
\hline Protein ID & TF Family & Identities and comments & References \\
\hline SAUSA300_0063 & Crp & Present in the ACME element & [9] \\
\hline SAUSA300_0093 & LysR & $\begin{array}{l}\text { YwqM (30.2\%) and GltR (27.9\%), in B. subtilis. The latter appears to be involved in glutamate } \\
\text { synthase expression. }\end{array}$ & [10] \\
\hline SAUSA300_0095 & LysR & $\begin{array}{l}\text { PtxR of Pseudomonas aeruginosa PAO1 (41.5\%) and Yersinia pestis (38.7\%). Activates the } \\
\text { expression of exotoxins and represses the expression of quorum sensing related genes. }\end{array}$ & {$[11,12]$} \\
\hline SAUSA300_0104 & AraC & Btr (24.5\%) from B. subtilis. One-component regulator that controls siderophore transport & [13] \\
\hline SAUSA300_0137 & GntR & $\begin{array}{l}\text { TreR (37\%), involved in the regulation of trehalose related genes in B. subtilis. It is encoded } \\
\text { divergent to purine synthesis genes. }\end{array}$ & [14] \\
\hline SAUSA300_0217 & TCS-RR & $\begin{array}{l}\text { YesN (38.4\%) and DegU (35.8\%) from B. subtilis. The latter is involved in the expression of } \\
\text { proteases and biofilm. }\end{array}$ & [15] \\
\hline SAUSA300_0238 & $B g \mid G$ & MnaR (25\%) from B. subtilis & \\
\hline SAUSA300_0258 & GntR & $\begin{array}{l}\text { LutR }(44.4 \%) \text {, involved in regulation of lactate and biofilm in B. subtilis. It has a UbiC } \\
\text { transcription regulator-associated (UTRA) domain. }\end{array}$ & {$[16]$} \\
\hline SAUSA300_0333 & BglG & $\begin{array}{l}\text { LicR }(28.5 \%) \text { from B. subtilis. Regulates the transport and degradation of oligomeric } \\
\text { beta-glucosides }\end{array}$ & [17] \\
\hline SAUSA300_0350 & Xre & YgzD (46\%) B. subtilis & \\
\hline SAUSA300_0373 & Xre & No identity to characterized proteins & \\
\hline SAUSA300_0503 & GntR & $\begin{array}{l}\text { YdeL (36\%) and GabR (32.3\%) both from B. subtilis. GabR regulates the expression of GABA } \\
\text { synthesis genes. It also has some identity to S. aureus NorG ( } 23 \%) \text {. It has a pyridoxal } \\
\text { phosphate (PLP)-dependent aspartate aminotransferase domain. }\end{array}$ & {$[18]$} \\
\hline SAUSA300_0577 & GntR & $\begin{array}{l}\text { This is the first gene in a putative operon with a pyridine nucleotide-disulphide } \\
\text { oxidoreductase. }\end{array}$ & \\
\hline SAUSA300_0658 & LysR & $\begin{array}{l}\text { E. coli OxyR ( } 29.4 \%) \text {, positive regulator for a hydrogen peroxide-inducible regulon. Possible } \\
\text { CcpC homolog, involved in regulation of TCA. }\end{array}$ & [19] \\
\hline SAUSA300_0683 & GntR & $\begin{array}{l}\text { IoIR (30.9\%), repressor or the myo-inositol operon in B. subtilis. Its genomic context shows } \\
\text { that it may regulate genes involved in fructose metabolism. }\end{array}$ & {$[20]$} \\
\hline SAUSA300_0803 & Xre & $\begin{array}{l}\text { Toxin-antitoxin systems. These systems may contribute to the preservation of plasmids and } \\
\text { genetic islands, however the role of many of them is still unknown }\end{array}$ & [21] \\
\hline SAUSA300_0804 & Xre & Toxin-antitoxin system & [21] \\
\hline SAUSA300_0878 & LysR & $\begin{array}{l}\text { CytR }(25 \%) \text {, regulator of the citrate synthase genes in B. subtilis. In S. aureus it is divergent to } \\
\text { isopropylmalate synthase involved in Leu and pyruvate metabolism. }\end{array}$ & {$[22]$} \\
\hline SAUSA300_0858 & Rps1 & B. subtilis YabR (42\%), putative polyribonucleotide nucleotidyl transferase & \\
\hline SAUSA300_0928 & Comk & B. subtilis ComK (33.9\%), required for genetic competence & [23] \\
\hline SAUSA300_0954 & MarR & YdgJ (35.4\%), B. subtilis & \\
\hline SAUSA300_0998 & Xre & Rpc (33.7\%) from B. subtilis bacteriophage phi 105 . Involved in the regulation of lysogeny. & \\
\hline SAUSA300_1170 & GntR & YmfC (34.3\%), B. subtilis. It has a UbiC transcription regulator-associated (UTRA) domain. & \\
\hline SAUSA300_1174 & GntR & YmfK (65\%), B. subtilis & \\
\hline SAUSA300_1175 & GntR & YmfM (31.25\%) B. subtilis & \\
\hline SAUSA300_1204 & Xre & No identity to characterized proteins & \\
\hline SAUSA300_1220 & TCS-RR & $\begin{array}{l}\text { LuxR-like protein with identity to DesR (43.2\%), responsible for thermosensing and signal } \\
\text { transduction at low temperatures in B. subtilis. Also has identity to YvfU (45\%) from B. subtilis }\end{array}$ & [24] \\
\hline SAUSA300_1424 & Unknown & No identity to characterized proteins & \\
\hline SAUSA300_1433 & Xre & No identity to characterized proteins & \\
\hline SAUSA300_1434 & Xre & Toxin-antitoxin system & [21] \\
\hline SAUSA300_1455 & AraC & AarP (30.8\%), involved in regulation of 2'-N-acetyltransferase in Providencia stuartii. & [25] \\
\hline SAUSA300_1469 & $\operatorname{Arg} R$ & $28 \%$ identity with S.aureus ArgR. In operon with a DNA repair protein & \\
\hline SAUSA300_1914 & GltR & $\begin{array}{l}\text { B. subtilis YtrA ( } 39.45 \%) \text {, possible repressor of an operon for a putative ATP-binding cassette } \\
\text { transport system involved in acetoin utilization. YtrA is an additional regulator of cell } \\
\text { envelope stress responses in B. subtilis. }\end{array}$ & {$[26,27]$} \\
\hline SAUSA300_1946 & $\operatorname{Rin} B$ & RinB (76\%) from phage 11. Activates int gene expression & [28] \\
\hline
\end{tabular}


Table 1 Possible role for uncharacterized TFs in S. aureus USA300 (Continued)

\begin{tabular}{|c|c|c|c|}
\hline SAUSA300_1968 & Xre & No identity to characterized proteins & \\
\hline SAUSA300_1969 & Xre & LexA (28\%), SOS regulator in E. coli & \\
\hline SAUSA300_2077 & $H \times I R$ & $\begin{array}{l}\text { B. subtilis YodB ( } 38.46 \%) \text {, regulation of yod (azoR1) after exposure to thiol-reactive compounds. } \\
\text { A similar gene in B. subtilis regulates formaldehyde detoxification via } h x \mid A B \text {. In } S \text {. aureus it is not } \\
\text { close to these genes, even though they are present in the genome. }\end{array}$ & [29] \\
\hline SAUSA300_2106 & BglG & ManR_(23.6\%), mannose utilization in B. subtilis & [30] \\
\hline SAUSA300_2160 & MerR & AdhR (38\%) B. subtilis. Transcriptional regulator involved in the response to aldehyde stress. & [31] \\
\hline SAUSA300_2216 & MarR & YwoH (31.6\%) from B. subtilis & \\
\hline SAUSA300_2248 & AraC & $\begin{array}{l}\text { E. coli YijO ( } 28.6 \%) \text {, might be involved in the regulation of genes encoding enzymes related } \\
\text { to PTS systems }\end{array}$ & [32] \\
\hline SAUSA300_2261 & GntR & No identity to characterized proteins & \\
\hline SAUSA300_2300 & TetR & $\begin{array}{l}\text { No identity to characterized proteins. Divergent to } 2 \text { multidrug transport proteins } \\
\text { (emrAB homologs) }\end{array}$ & \\
\hline SAUSA300_2310 & LytTr & Bears a LytTR domain, which is an only recently characterized family. & \\
\hline SAUSA300_2322 & TetR & B. subtilis YxbF (42.4\%). In S. aureus it is in an operon with a CorA Mg transporter & \\
\hline SAUSA300_2336 & MerR & CueR (42.8\%), involved in copper induction in B. subtilis. & [33] \\
\hline SAUSA300_2445 & MerR & $\begin{array}{l}36 \% \text { identical to BltR, B. subtilis, and MerR ( } 31 \%) \text {, S. aureus. The former is involved in response } \\
\text { to structurally dissimilar drugs, while the latter is on a plasmid specifying resistance for } \\
\text { mercurial compounds. }\end{array}$ & {$[34,35]$} \\
\hline SAUSA300_2547 & Unknown & B. subtilis YuaC (55.4\%) & \\
\hline SAUSA300_2452 & MarR & $\begin{array}{l}\text { Similar to B. subtilis YvnA (35.8\%), (29\%) and AdcR from Streptococcus pneumoniae. AdcR is } \\
\text { able to sense metals for the regulation of zinc uptake proteins related genes encoding } \\
\text { cell-surface zinc-binding pneumococcal histidine triad proteins and AdcAll (laminin binding). } \\
\text { Also has a 33\% identity to SarZ }\end{array}$ & [36] \\
\hline SAUSA300_2459 & MarR & $\begin{array}{l}\text { MhqR (41.5\%) regulates multiple dioxygenases/glyoxalases and an azoreductase that confer } \\
\text { resistance to 2-methylhydroquinone and catechol in B. subtilis }\end{array}$ & [37] \\
\hline SAUSA300_2490 & LysR & $\begin{array}{l}\text { No identities to characterized proteins. Divergent to operon encoding } \mathrm{mmpL} \text { (transporter) } \\
\text { and Feo iron dependent transporters }\end{array}$ & \\
\hline SAUSA300_2509 & TetR & B. subtilis YxbF (31.6\%). & \\
\hline SAUSA300_2515 & TetR & $\begin{array}{l}\text { SImA (26.2\%) in Vibrio parahaemolyticus. SImA proteins are involved in nucleoid occlusion } \\
\text { systems in E. coli. In S. aureus it is in an operon with genes encoding an oxidoreductase, } \\
\text { an amidohyrolase and a hydrolase. }\end{array}$ & [38] \\
\hline SAUSA300_2530 & TetR & No identity to characterized proteins. & \\
\hline SAUSA300_2563 & MarR & PetP, (33.06\%), necessary for photosynthetic and respiratory growth in Rhodobacter capsulatus & [39] \\
\hline SAUSA300_2575 & BglG & No identity to characterized proteins. & \\
\hline SAUSA300_2640 & Xre & ImmR (46\% identity), involved in mobilization of the genetic element ICEB1 in B. subtilis & {$[40,41]$} \\
\hline SAUSA300_2625 & PadR & PadR (37.5\%), repressor of phenolic acid response genes in B. subtilis & [42] \\
\hline
\end{tabular}

Amino acid sequences of uncharacterized TFs (Figure 2) were analyzed by using BLAST comparisons against the NR and SwissProt databases. In the third column is shown the closest identified protein (s), and their functional roles in corresponding organisms.

appears to have occurred, such as in Dictyoglomus turgidum and Bifidobacterium animalis ssp. animalis, amongst others (reviewed in [43]). Examples of these proteins are SAUSA300_1632 (transcriptional repressor of ribonucleotide reductases genes, NrdR) and $\mathrm{HrcA}$ (SAUSA300_1542), a regulator of genes involved in heatshock. The third group of proteins was identified as being conserved mainly in the Firmicutes phylum. This group includes NsaR, GraR, and AgrA, proteins that serve as the response regulator of two-component systems, GapR and TreR (GntR-like proteins involved in the regulation of metabolism related genes), GlnR (glutamine synthetase repressor), HutR (repressor of the histidine utilization operon, hut) and SarZ (which promotes the expression of virulence genes), as well as other as yet uncharacterized proteins. Group 4 includes TFs with very few homologues outside the Staphylococacceae family, and thus represents Staphylococacceae specific TFs. This group contains most of the members of the Sar family (Rot, SarA, SarV, SarY, SarR, SarU, SarT, SarS and SarX), the alternative sigma factor $\sigma^{S}$, and as yet uncharacterized TFs from the MarR, AraC/XylS and Xre families.

As such, by using the TFs found in S. aureus USA300 as a scaffold to interrogate other sequenced bacterial 


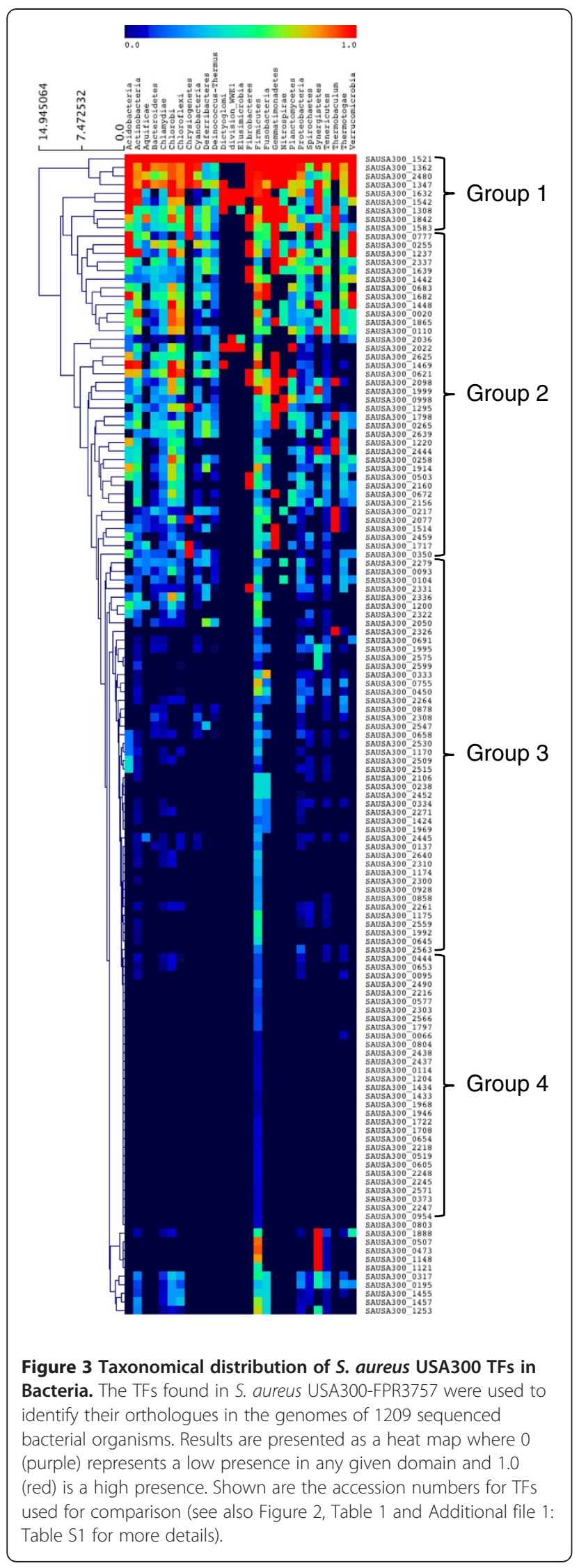

genomes, we were able to identify: (i) TFs that are conserved in all the bacterial phyla, suggesting an ancient origin and critical cellular function, (ii) those regulators found mainly in the Firmicutes, and (iii) regulators found exclusively in the Staphylococacceae.

\section{Comparison of TFs between S. aureus strains}

To further explore TFs conserved in the Staphylococacceae, and identify those that are potentially involved in the regulation of virulence gene expression, we compared the USA300 TFs with those in other Staphylococcal strains. Eleven additional $S$. aureus strains were examined (Additional file 1: Table S2); ten of which are human specific pathogens, while one (RF122) is a pathogen of cattle, and has important agricultural implications. These strains were selected as they have been extensively studied, and are representative of the wide genetic variability across $S$. aureus strains. We determined that the total number of TFs and $\sigma$ factors varies from strain to strain, ranging from 126 for the bovine pathogenic strain RF122, to 151 for the MRSA strain N315 (Figure 4). This variation among different strains is most likely the result of genomic rearrangements, duplications, and the acquisition of novel genetic elements, such as phages and pathogenicity islands [44].

A total of 112 TFs were identified as being present in all S. aureus strains, including regulators of genes involved in metabolic (ArcR, Fur, HutR, GntR, GlnR, CcpA, ArgR, ArcR, FeoA, Fur, PerR, FemC, TreR, GapR, LacR, FapR, CcpA, PurR, HisR, and multiple TCS) and virulence processes (AgrA, SarA, SarR, SarS, SarV, SarX, SarY, SarZ, Rot and MgrA). The high degree of conservation of these TFs probably emphasizes the need for specific and precise regulation of genes involved in these key physiological processes. In addition, we found TFs in this group that are associated with genome homeostasis such as LexA and HU that respond to DNA damage and structure, respectively. Unsurprisingly, given their role in transcription processes, all the $\sigma$ factors $\left(\sigma^{\mathrm{A}}, \sigma^{\mathrm{B}}, \sigma^{\mathrm{H}}\right.$ and $\sigma^{\mathrm{S}}$ ) were conserved across all strains.

Conversely, a number of TFs were found in most strains, but were absent in one or two. The absence of these TFs suggests that their loss leads to a difference in the strain specific regulation of important pathways. Amongst this group are the TCS-RRs. Fourteen of the sixteen $S$. aureus TCS are conserved in all strains analyzed, however ArlR, for example, is present, yet truncated, in strain N315. Similarly, the uncharacterized SAUSA300_1220 is truncated in strain MRSA252; and both SAUSA300_1220 and SrrA are absent from the bovine pathogen RF122 (Figure 2). Interestingly, for these latter two regulators, the sensor histidine kinase is also missing. Additionally, a rare event occurs where the TCS KdpDE is duplicated on SCCmec II; thus strains N315, 


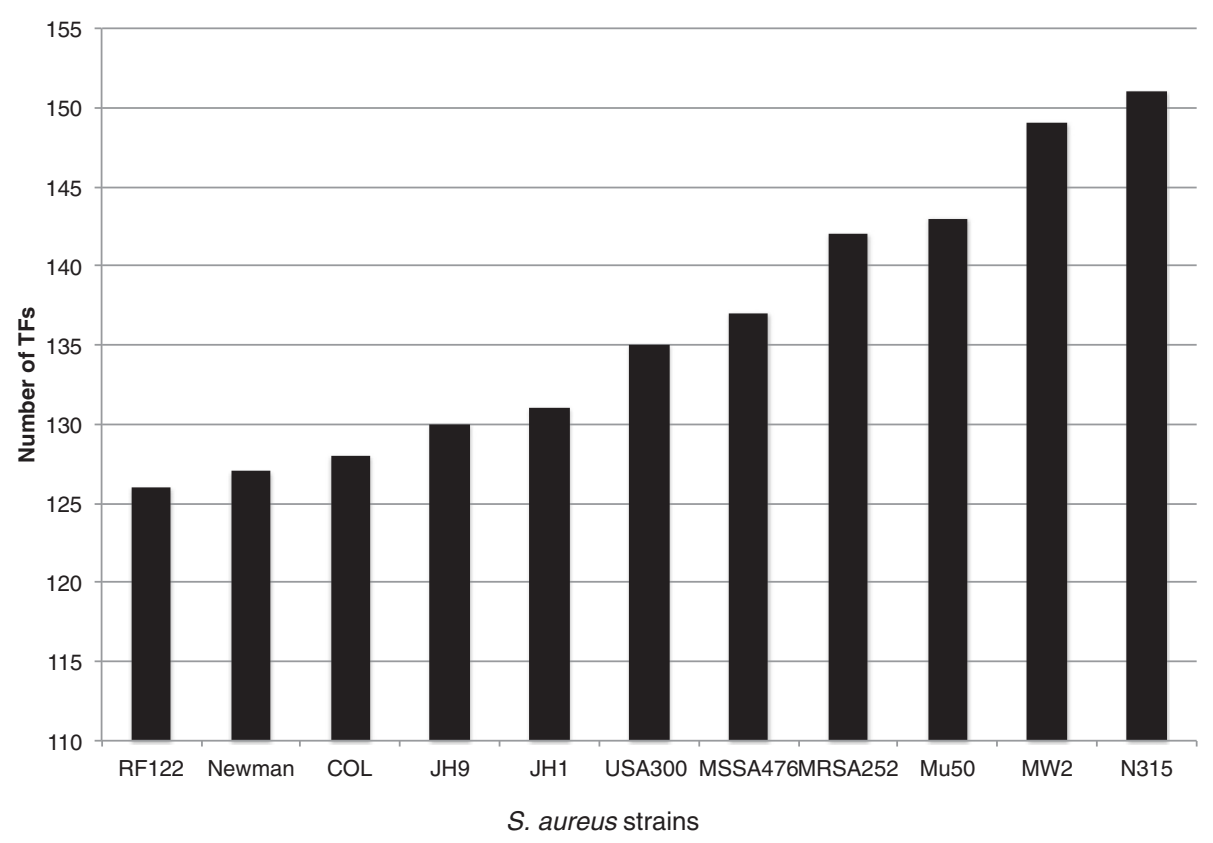

Figure 4 Total number of TFs in multiple S. aureus strains. TFs in all S. aureus strains were detected using a similar method to that used for strain USA300-FPR-3757.

Mu50, JH1, JH9, Mu3 and MRSA252 are unique in carrying two copies of this regulatory system. The occurrence of this duplication has previously been observed [45], however its biological significance is not yet clear.

In the context of non-TCS-RR, other TFs are also variable across S. aureus strains, including SAUSA300_0063, which is only found in USA300. This TF is a likely duplication of the ArgR arginine repressor, and is encoded on the arginine catabolic mobile element (ACME), which is present only in USA300 strains and is linked to SCCmec IV [46]. By far, the most variability within a family of TFs was observed for the Xre-like elements. This family includes regulators in Eukaryotes, Archaea and Bacteria, and is evolutionarily related to the bacteriophage regulators Cro and cI [47]. Our analysis showed that $S$. aureus USA300-FPR3757 has $13 \mathrm{pu}-$ tative members of this family. To our knowledge XdrA, which is present in all strains analyzed and serves as an activator of the virulence factor protein A [48], is the only member of this family that has been characterized. In contrast to some of the Xre-like proteins found herein, XrdA is not encoded on or near a phage-related element. Other Xre regulators also exist that are similarly unassociated with lysogenic bacteriophages, including SAUSA300_0804, SAUSA300_2640 and SAUSA300_0998. In total, five Xrelike TFs were found in all S. aureus strains, and appear to be unassociated with phage-like elements. It is tempting to suggest that the presence or absence of these Xre elements could be considered a genetic fingerprint for each of the strains, and may influence regulatory network in subtle yet wide-reaching ways.

\section{Discussion}

The overall aim of this study was to gain insight into the composition and conservation of TFs in the Staphylococacceae, specifically in the major human pathogen, S. aureus. First we detected TFs in the USA300-FPR3757 strain, identifying 135 elements belonging to 36 different regulatory families. Of note, almost half of these (58 out of 135 , or $42.9 \%$ ) have yet to be characterized. Herein we were able to propose possible roles for most of them, leaving only 9 without ascribed or predicted functions.

The most abundant TFs in this strain belonged to the MarR family, which includes the Sar-like subfamily [49]. One such TF (SAUSA300_2452) was of particular interest as it showed 33\% identity with SarZ over $64 \%$ of the length of the protein, as determined using BLASTP, suggesting that it might be a new member of this family. In order to corroborate whether this protein is related to the Sar family we generated a phylogenetic tree with all known Sar and MarR proteins found in the USA300 strain and compared them with the crystal structure of MgrA. As seen in Additional file 2: Figure S1, MarR-like TFs were grouped in three clades: one including SarX, TcaR and four non-characterized MarR proteins; a second included SarA, SarY, SarR, SarS, SarU, SarV and Rot; and the third included MgrA, SarZ and SAUSA300_2452. From this analysis it seems that SAUSA300_2452 is phylogenetically related to SarZ and MgrA, suggesting it may belong to this subfamily. 
Given the adaptability of $S$. aureus to multiple environments it is perhaps no surprise to find that TCSRR family was one of the most abundant families, with 16 members. Despite the fact that this group of proteins has been widely studied, two members remain uncharacterized, SAUSA300_1220 and SAUSA300_0217. SAUSA300_1220 shares homology with B. subtilis DesR (43\% identity over $99 \%$ of the protein, as determined using BLASTP), which is involved in sensing changes in temperature and regulating the expression of genes that respond to this environmental cue [24]. Of note, in gamma-proteobacteria this role is accomplished by the histone-like protein $\mathrm{H}-\mathrm{NS}$ and other related factors, which are seemingly absent in the firmicutes [50]. Indeed, in $S$. aureus there is only one protein related to the histone-like family, suggesting that regulation of the thermal response is achieved by other TFs, which may include SAUSA300_1220. SAUSA300_0217 has some identity to DegU (35.8\% identity over $42 \%$ of the protein, as determined using BLASTP) from B. subtilis, which is involved in the modulation of protease expression and biofilm formation. Importantly, there is some suggestion that this system, or rather its counterpart in strain COL, is expressed during anaerobiosis [51].

We also used the USA300 TFs as a scaffold to define how conserved these regulators are within eubacterial species. This analysis showed that nine TFs have orthologues in almost all of the 1209 genomes analyzed. This suggests that these proteins have an important role in cell fitness, and have a common ancestral origin. Included in this group are: SAUSA300_1521, the primary sigma factor $\sigma^{70}\left(\sigma^{\mathrm{A}}\right)$ that drives house-keeping gene expression; and the histone-like protein $\mathrm{HU}$ (SAUSA300_1362), which is important in controlling DNA structure [50]. Also included in this group is SAUSA300_1347 (BirA), which is involved in regulation and biotinylation of the essential metabolic factor CoA [52]. CidR (SAUSA300_2480) regulates the expression of holin/anti-holin complexes involved in peptidoglycan synthesis, and is therefore important for bacterial survival, at least in S. aureus strains [53]. SAUSA300_1632 is a $\mathrm{NrdR}$ orthologue that regulates the expression of ribonucleotide reductases, necessary for DNA and RNA synthesis [54]. HrcA (SAUSA300_1542) is an important regulator of proteins involved in the heat-shock response in Bacillus subtilis [55]. Though less conserved than the other TFs in group 1, ArlR (SAUSA300_1308) is still preserved in many bacterial phyla. ArlR is a twocomponent response regulator that controls the expression of 114 genes in S. aureus, including those involved in cell division and growth [56].

We also identified a group of regulatory proteins whose orthologues are conserved within most Firmicutes, and are involved in processes such as metabolism (GapR,
TreR, GlnR, HutR, Hex, RpiRC, ScrR), stress response (NsaR, GraR, MepR) and virulence (AgrA, SarZ, SaeR, IcaR, Rsp). The prevalence of TFs related to metabolism and stress in so many Firmicutes would be expected as this suggests a common origin. It is interesting to note that while highly conserved regulators are involved in key cellular processes, TFs that are phylum specific are involved in more specialized functions i.e. stress response and virulence. For example, it is possible that in nonpathogenic organisms, those TFs known to regulate virulence genes in other species serve to control genes for niche adaptation or symbiosis.

At the most specific level, we defined those TFs that were conserved uniquely in the Staphylococacceae. Most of the TFs in this group are related to virulence and environment adaptation, including the Sar family of proteins, the alternative sigma factor SigS, and some elements involved in metabolism. Collectively, and to our knowledge, this is the first global study that circumscribes the TFs for the Firmicutes, and more specifically, the Staphylococacceae.

Another of our objectives was to define how conserved TFs are across multiple, well-characterized S. aureus strains. We first identified the TFs for eleven additional strains (Figure 4, and data not shown) and then compared them with those in USA300. The majority of the TFs were conserved across all strains (83\%), which is largely comprised of those that are part of the core Staphylococcal TF suite. The absence of the other $17 \%$ of regulatory proteins indicates that these are not central for survival or pathogenesis, and may be responsible for subtle, strain specific, fine-tuning of gene expression patterns. For instance, SAUSA300_0063 is a Crp-like TF encoded in the ACME region. ACME has been found only in USA300 strains, and is thought to play a role in virulence [46,57]. All known USA300 strains have this genetic element, supporting its role in virulence processes and/or transmission. In contrast, Rbf, an AraC-like protein that positively regulates biofilm formation [58] is present in all strains except MRSA252. This exemplifies our contention that differences in regulatory networks adapt to strain specific process. For example, MRSA252 is a robust biofilm forming isolate, yet is still capable of undergoing this process in the absence of Rbf. This suggests that this process is multifactorial, involving many different regulators, and is adaptable within strains to individual growth or pathogenic environments.

The biggest difference in TFs amongst Staphylococcal strains was observed in phage related regulators. These demonstrated the most variability, which is unsurprising, as each strain has acquired variable phage content over time. Despite the fact that they are located on phage elements, some have developed a key role in the regulation of virulence genes in the core chromosome. Such is 
the case of XdrA, which regulates the expression of protein $\mathrm{A}$, an important immune evasion virulence factor. Moreover, some TFs are located in the vicinity of putative toxin-antitoxin systems, e.g. SAUSA300_2640; such systems have been suggested to contribute to the preservation of plasmids and genetic islands [21]. Additionally, one of these TFs (SAUSA300_0998) is located close to a putative putrescine secretion system, possibly forming an operon, suggesting it might be involved in its regulation.

We also compared TF variation from human-specific S. aureus strains to the bovine adapted isolate RF122, which is associated with mastitis in cattle. Such strains are of significant environmental and economic importance as they are responsible for massive losses financially in animal production each year [59]. We hypothesized that the difference in host preference for RF122 would coincide with altered TF content when compared to human adapted strains of $S$. aureus. A comparison of the TFs from USA300 with those in RF122 identified 17 elements that were unique to human versus bovine strains (Table 2). While it is difficult to predict the combined outcome on gene expression this variation would have, certain examples suggest changes to virulence gene expression in RF122 that may account for altered species specificity. An example of this is the loss of SarT and SarU in RF122 (quoted in [60]). SarT influences the expression of sarS, and hence that of spa, while at the same time repressing the expression of $\alpha$-toxin (hla), sarU and agr [61,62]. Hla is a central virulence factor, known to be important for infection in animal models of disease causation [63]. Thus, given the absence of SarT, it is possible that Hla is upregulated in RF122. In support of this, a recent study of multiple bovine $S$. aureus isolates, including RF122, revealed increased production of Hla [64]. Indeed, it was shown that overexpression of hla was not only due to the presence of SNPs, but also upregulation by SarZ, and possibly by the elevated expression of other regulators such as AgrA, SaeR and ArlR. An additional 9 TFs were identified in RF122 that were not present in USA300 (Additional file 1: Table S1). Many of these are Xre family proteins, and are associated with horizontally acquired DNA. Studies have previously shown that RF122 has a distinctive pathogenicity island (SaPIbov) [65], therefore it is possible that these horizontally acquired TFs, together with additional virulence genes in RF122, form a regulatory network that governs host specificity. As we have demonstrated here, although RF122 lacks some TFs, which in turn has the capacity to render it more virulent, it also has additional TFs that could potentially regulate well-known virulence genes, and maybe those in strainspecific genetic regions such as SaPIbov.
Table 2 TFs specific to USA300 and RF122

\begin{tabular}{ll}
\hline TFs present only in RF122 & USA300 TFs absent in RF122 \\
\hline SAB2083C & SrrA \\
SAB1911 & SAUSA300_1220 \\
SAB1910 & SAUSA300_1424 \\
SAB1750C & SAUSA300_0093 \\
SAB1757 & SAUSA300_0954 \\
SAB1836C & SAUSA300_0095 \\
SAB1297 & SAUSA300_0238 \\
SAB1256C & SAUSA300_2452 \\
& SAUSA300_0858 \\
& SarT \\
& SarU \\
& SAUSA300_1434 \\
& SAUSA300_0804 \\
& SAUSA300_0803 \\
& SAUSA300_1968 \\
& SAUSA300_1433 \\
& SAUSA300_1969
\end{tabular}

\section{Conclusions}

In summary, the analysis presented herein demonstrates the incredible complexity of regulatory networks and gene regulation in S. aureus, and offers unique insights into many as yet uncharacterized TFs in this important human pathogen. A comparison of S. aureus TFs with those of other bacterial phyla reveals two main types of TF in Staphylococci. The first group represents a core of regulators, present in common ancestors of diverse bacteria that participates in the regulation of key cellular processes. The second group represents TFs whose function seems to be genus/species specific (e.g. virulence gene regulators and those for specific metabolic requirements). Therefore we propose that TFs in group 4 forms the core set of TFs in the Staphylococcaceae. Included in this group are most the Sar regulators, which are part of the MarR family, and other, as yet uncharacterized proteins. Additionally, we focused on the differences amongst well-characterized $S$. aureus strains and found absence of TFs that might dictate changes in regulatory networks for each isolate. Finally, the similarities and differences in TF content between the human pathogen USA300 and the bovine pathogen RF122 were determined. Previous reports have shown that the expression of virulence factors amongst bovine and human isolates is different, and here we observed differences in the TFs content for these two strains. It is possible that some of these elements are involved in differentially regulating virulence factors, perhaps through modulation of known elements such as AgrA and SarA. 


\section{Methods}

\section{Identification of DNA-binding transcription factors}

The complete genomes of twelve $S$. aureus strains were obtained from ftp://ftp.ncbi.nlm.nih.gov/genomes/ Bacteria. Open reading frames that encode predicted protein sequences, i.e. the proteome in all bacteria, were considered as annotated genes. In order to identify the repertoire of TFs in $S$. aureus strains, domain assignations associated to DNA-binding regions in the Superfamily database (25-Apr-2010 version), and others identified and annotated in PFAM [66] were used. Additionally, family-specific Hidden Markov Models (HMM) constructed from three bacterial models: Escherichia coli K-12, Bacillus subtilis, and Corynebacterium glutamicum were used to search $S$. aureus genomes. Briefly, 90 family-specific HMMs previously reported for E. coli K-12 [67] and 57 family-specific HMMs from B. subtilis [68] were used to scan complete genome sequences (E-value threshold of $10^{-3}$ ), with the "hmmsearch" module from the HMMer suite of programs (http://hmmer.janelia.org/). These HMMs were constructed using the previously identified $\mathrm{TF}$ families in E. coli $\mathrm{K}-12$ and B. subtilis as seeds, considering the DNA-binding domain (DBD) sequence (around 60 amino acids) of every protein from multiple families. S. aureus USA300 proteome sequences were scanned with these HMMs, and proteins with less than $60 \%$ coverage in the DNAbinding region against their corresponding HMM were excluded. Finally, regulators deposited in the DBD database [69] were also considered as potential DNAbinding TFs.

In order to evaluate the distribution of TFs and their corresponding orthologues across all bacterial genomes, a hierarchical average linkage-clustering algorithm was applied with a Manhattan correlation distance as a similarity measure. Analyses were performed using the program Mev4 (multi-experiment viewer; PMID:12613259). In order to determine the relative abundance of TFs and their orthologues, we calculated the fraction of genomes in the group that had at least one member versus the number of representative organisms. Thus, the following formula was considered: relative abundance by phylum (total number of orthologues identified)/(total number of organisms by phylum). Thus, a value of 1 corresponds to presence and 0 represents absence. Because our aim was to evaluate the taxonomical distribution of orthologues proteins, 27 taxonomical phyla corresponding to eubacteria were considered.

In order to achieve comparative analysis strain USA300FPR3757 was used for the classification of TFs into evolutionary families. This was based on PFAM annotations, and corroborated using BLAST searches (using default conditions) against well-annotated protein families.

\section{Comparison of ORFomes from different S. aureus strains} Based on the USA300-FPR3757 ORFome, we searched for the presence and absence of TFs in eleven different strains, including: Newman, COL, JH1, JH9, MW2, Mu50, Mu3, N315, RF122, MRSA252 and MSSA476. This comparison was achieved by sequence analysis using the Comprehensive Microbial Resources (CRM) database from JCVI (http://cmr.jcvi.org/tigr-scripts/CMR/CmrHomePage.cgi), and confirmed by BLAST searches.

Additionally, in order to evaluate the phylogenetic distribution, the $S$. aureus TF repertoire was used to identify orthologous proteins in 1209 sequenced eubacterial strains. Orthologous relationships were identified based on BLASTP reciprocal best hits, with an E-value cut-off of $\leq 1 \mathrm{e}-6$, as described elsewhere [70]. Finally, the phylogenetic distribution of each TF was evaluated based on a hierarchical cluster analysis.

\section{Additional files}

Additional file 1: Table S1. S. aureus USA300 TFs conserved in eubacteria. Groups are defined as: 1, proteins with orthologues in $60-100 \%$ of genomes studied; 2, 15-59\%; 3, 1-14\%; and $4<1 \%$ of genomes. Table S2. S. aureus strains analyzed in this work.

Additional file 2: Figure S1. Phylogenetic tree for the MarR-like proteins. Sar and MarR proteins, along with uncharecterized MarR proteins, were aligned using the MgrA crystal (2BV6) structure as a template. Three clades are denoted, see text for details. SarU, SAUSA300_1114 and SAUSA300_2247 exhibit two MgrA-like domains and therefore are shown as duplicated in the tree.

\section{Competing interests}

The authors declare that they have no competing interests.

\section{Authors' contributions}

JAI and EP-R conceived the study and its coordination. EP-R performed all the bioinformatics analysis, including large-scale sequence analysis; JAI helped in bioinformatics analysis and data mining. RKC and LNS helped in the design of the study. JAI, EP-R, RKC and LNS drafted and wrote the manuscript. All authors read and approved the final manuscript.

\section{Acknowledgements}

This work was supported by grants IN-209511 (EP-R) from DGAPA-UNAM, 155116 (EP-R) from the National Council for Science and Technology (CONACYT) and Al080626 (LNS) from the National Institute of Allergies and Infectious Disease.

\section{Author details}

${ }^{1}$ Department of Cell Biology, Microbiology and Molecular Biology, University of South Florida, 4202 East Fowler Avenue, ISA 2015, Tampa, FL 33620-5150, USA. ${ }^{2}$ Departamento de Ingeniería Celular y Biocatálisis, Instituto de Biotecnologia UNAM, Av Universidad 2001, Cuernavaca, Morelos CP 62210, Mexico. ${ }^{3}$ Present address: Departamento de Microbiología, Escuela Nacional de Ciencias Biológicas, Instituto Politécnico Nacional, Prol. De Carpio y Plan de Ayala. Col. Sto. Tomás, México, DF CP 11340, Mexico.

Received: 21 October 2012 Accepted: 12 February 2013 Published: 26 February 2013

\section{References}

1. Kobayashi SD, DeLeo FR: An update on community-associated MRSA virulence. Curr Opin Pharmacol 2009, 9(5):545-551. 
2. David MZ, Daum RS: Community-associated methicillin-resistant Staphylococcus aureus: epidemiology and clinical consequences of an emerging epidemic. Clin Microbiol Rev 2010, 23(3):616-687.

3. Holden MT, Feil EJ, Lindsay JA, Peacock SJ, Day NP, Enright MC, Foster TJ, Moore CE, Hurst L, Atkin R, et al: Complete genomes of two clinical Staphylococcus aureus strains: evidence for the rapid evolution of virulence and drug resistance. Proc Natl Acad Sci U S A 2004, 101(26):9786-9791.

4. Rolain JM, Francois P, Hernandez D, Bittar F, Richet H, Fournous G, Mattenberger Y, Bosdure E, Stremler N, Dubus JC, et al: Genomic analysis of an emerging multiresistant Staphylococcus aureus strain rapidly spreading in cystic fibrosis patients revealed the presence of an antibiotic inducible bacteriophage. Biol Direct 2009, 4:1

5. Thurlow $L R$, Joshi GS, Richardson AR: Virulence strategies of the dominant USA300 lineage of community-associated methicillin-resistant Staphylococcus aureus (CA-MRSA). FEMS Immunol Med Microbiol 2012, 65(1):5-22.

6. Witte W: Community-acquired methicillin-resistant Staphylococcus aureus: what do we need to know? Clin Microbiol Infect 2009, 15(Suppl 7):17-25.

7. Tenover FC, McDougal LK, Goering RV, Killgore G, Projan SJ, Patel JB, Dunman PM: Characterization of a strain of community-associated methicillin-resistant Staphylococcus aureus widely disseminated in the United States. J Clin Microbiol 2006, 44(1):108-118.

8. Li M, Diep BA, Villaruz AE, Braughton KR, Jiang X, DeLeo FR, Chambers HF, LU Y, Otto M: Evolution of virulence in epidemic community-associated methicillin-resistant Staphylococcus aureus. Proc Natl Acad Sci USA 2009, 106(14):5883-5888.

9. Goering RV, McDougal LK, Fosheim GE, Bonnstetter KK, Wolter DJ, Tenover FC: Epidemiologic distribution of the arginine catabolic mobile element among selected methicillin-resistant and methicillin-susceptible Staphylococcus aureus isolates. J Clin Microbio/ 2007, 45(6):1981-1984.

10. Belitsky BR, Sonenshein AL: Altered transcription activation specificity of a mutant form of Bacillus subtilis GltR, a LysR family member. J Bacteriol 1997, 179(4):1035-1043.

11. Carty NL, Layland N, Colmer-Hamood JA, Calfee MW, Pesci EC, Hamood AN: PtxR modulates the expression of QS-controlled virulence factors in the Pseudomonas aeruginosa strain PAO1. Mol Microbiol 2006, 61(3):782-794.

12. Colmer-Hamood JA, Aramaki H, Gaines JM, Hamood AN: Transcriptional analysis of the Pseudomonas aeruginosa toxA regulatory gene $p t x R$. Can J Microbiol 2006, 52(4):343-356.

13. Gaballa A, Helmann JD: Substrate induction of siderophore transport in Bacillus subtilis mediated by a novel one-component regulator. Mol Microbiol 2007, 66(1):164-173.

14. Schock F, Dahl MK: Expression of the tre operon of Bacillus subtilis 168 is regulated by the repressor TreR. J Bacteriol 1996, 178(15):4576-4581.

15. Mader U, Antelmann H, Buder T, Dahl MK, Hecker M, Homuth G: Bacillus subtilis functional genomics: genome-wide analysis of the DegS-DegU regulon by transcriptomics and proteomics. Mol Genet Genomics 2002, 268(4):455-467.

16. Chai $Y$, Kolter $R$, Losick R: A widely conserved gene cluster required for lactate utilization in Bacillus subtilis and its involvement in biofilm formation. J Bacteriol 2009, 191(8):2423-2430.

17. Tobisch S, Stulke J, Hecker M: Regulation of the lic operon of Bacillus subtilis and characterization of potential phosphorylation sites of the LicR regulator protein by site-directed mutagenesis. J Bacterio/ 1999, 181(16):4995-5003.

18. Belitsky BR, Sonenshein AL: GabR, a member of a novel protein family, regulates the utilization of gamma-aminobutyrate in Bacillus subtilis. Mol Microbiol 2002, 45(2):569-583.

19. Tao K, Makino K, Yonei S, Nakata A, Shinagawa H: Purification and characterization of the Escherichia coli OxyR protein, the positive regulator for a hydrogen peroxide-inducible regulon. J Biochem 1991, 109(2):262-266.

20. Yoshida Kl, Aoyama D, Ishio I, Shibayama T, Fujita Y: Organization and transcription of the myo-inositol operon, iol, of Bacillus subtilis. J Bacteriol 1997, 179(14):4591-4598

21. Van Melderen $L$, Saavedra De Bast M: Bacterial toxin-antitoxin systems: more than selfish entities? PLoS Genet 2009, 5(3):e1000437.

22. Jin S, Sonenshein AL: Transcriptional regulation of Bacillus subtilis citrate synthase genes. J Bacteriol 1994, 176(15):4680-4690.

23. van Sinderen D, ten Berge A, Hayema BJ, Hamoen L, Venema G: Molecular cloning and sequence of comK, a gene required for genetic competence in Bacillus subtilis. Mol Microbiol 1994, 11(4):695-703.
24. Aguilar PS, Hernandez-Arriaga AM, Cybulski LE, Erazo AC, de Mendoza D: Molecular basis of thermosensing: a two-component signal transduction thermometer in Bacillus subtilis. EMBO J 2001, 20(7):1681-1691.

25. Macinga DR, Parojcic MM, Rather PN: Identification and analysis of aarP, a transcriptional activator of the 2'-N-acetyltransferase in Providencia stuartii. J Bacteriol 1995, 177(12):3407-3413.

26. Yoshida Kl, Fujita Y, Ehrlich SD: An operon for a putative ATP-binding cassette transport system involved in acetoin utilization of Bacillus subtilis. J Bacterio/ 2000, 182(19):5454-5461.

27. Salzberg LI, Luo Y, Hachmann AB, Mascher T, Helmann JD: The Bacillus subtilis GntR family repressor YtrA responds to cell wall antibiotics. J Bacteriol 2011, 193(20):5793-5801.

28. Ye $\mathrm{ZH}$, Lee $C Y$ : Cloning, sequencing, and genetic characterization of regulatory genes, $\operatorname{rin} A$ and $\operatorname{rin} B$, required for the activation of staphylococcal phage phi 11 int expression. J Bacteriol 1993, 175(4):1095-1102.

29. Leelakriangsak M, Huyen NT, Towe S, van Duy N, Becher D, Hecker M, Antelmann $H$, Zuber P: Regulation of quinone detoxification by the thiol stress sensing DUF24/MarR-like repressor, YodB in Bacillus subtilis. Mol Microbiol 2008, 67(5):1108-1124.

30. Sun T, Altenbuchner J: Characterization of a mannose utilization system in Bacillus subtilis. J Bacteriol 2010, 192(8):2128-2139.

31. Nguyen TT, Eiamphungporn W, Mader U, Liebeke M, Lalk M, Hecker M Helmann JD, Antelmann H: Genome-wide responses to carbonyl electrophiles in Bacillus subtilis: control of the thiol-dependent formaldehyde dehydrogenase AdhA and cysteine proteinase YraA by the MerR-family regulator YraB (AdhR). Mol Microbiol 2009, 71(4):876-894.

32. Ibarra JA, Perez-Rueda E, Segovia L, Puente JL: The DNA-binding domain as a functional indicator: the case of the AraC/XylS family of transcription factors. Genetica 2008, 133(1):65-76.

33. Gaballa A, Cao M, Helmann JD: Two MerR homologues that affect copper induction of the Bacillus subtilis copZA operon. Microbiology 2003, 149(Pt 12):3413-3421.

34. Laddaga RA, Chu L, Misra TK, Silver S: Nucleotide sequence and expression of the mercurial-resistance operon from Staphylococcus aureus plasmid pl258. Proc Natl Acad Sci USA 1987, 84(15):5106-5110.

35. Ahmed M, Lyass L, Markham PN, Taylor SS, Vazquez-Laslop N, Neyfakh AA: Two highly similar multidrug transporters of Bacillus subtilis whose expression is differentially regulated. J Bacteriol 1995, 177(14):3904-3910.

36. Reyes-Caballero H, Guerra AJ, Jacobsen FE, Kazmierczak KM, Cowart D, Koppolu UM, Scott RA, Winkler ME, Giedroc DP: The metalloregulatory zinc site in Streptococcus pneumoniae AdcR, a zinc-activated MarR family repressor. J Mol Microbiol 2010, 403(2):191-216.

37. Towe S, Leelakriangsak M, Kobayashi K, Van Duy N, Hecker M, Zuber P, Antelmann H: The MarR-type repressor MhqR (YkvE) regulates multiple dioxygenases/glyoxalases and an azoreductase which confer resistance to 2-methylhydroquinone and catechol in Bacillus subtilis. Mol Microbiol 2007, 66(1):40-54.

38. Tonthat NK, Arold ST, Pickering BF, Van Dyke MW, Liang S, Lu Y, Beuria TK, Margolin W, Schumacher MA: Molecular mechanism by which the nucleoid occlusion factor, SImA, keeps cytokinesis in check. EMBO J 2011, 30(1):154-164

39. Tokito MK, Daldal F: petR, located upstream of the $f b c F B C$ operon encoding the cytochrome bc1 complex, is homologous to bacterial response regulators and necessary for photosynthetic and respiratory growth of Rhodobacter capsulatus. Mol Microbiol 1992, 6(12):1645-1654.

40. Bose B, Auchtung JM, Lee CA, Grossman AD: A conserved anti-repressor controls horizontal gene transfer by proteolysis. Mol Microbiol 2008, 70(3):570-582

41. Auchtung JM, Lee CA, Garrison KL, Grossman AD: Identification and characterization of the immunity repressor (ImmR) that controls the mobile genetic element ICEBs1 of Bacillus subtilis. Mol Microbiol 2007, 64(6):1515-1528.

42. Nguyen TK, Tran NP, Cavin JF: Genetic and biochemical analysis of PadRpadC promoter interactions during the phenolic acid stress response in Bacillus subtilis 168. J Bacteriol 2011, 193(16):4180-4191.

43. Ventura M, O'Flaherty S, Claesson MJ, Turroni F, Klaenhammer TR, van Sinderen D, O'Toole PW: Genome-scale analyses of health-promoting bacteria: probiogenomics. Nat Rev Microbiol 2009, 7(1):61-71.

44. Novick RP, Christie GE, Penades JR: The phage-related chromosomal islands of Gram-positive bacteria. Nat Rev Microbiol 2010, 8(8):541-551. 
45. Ito T, Katayama Y, Asada K, Mori N, Tsutsumimoto K, Tiensasitorn C, Hiramatsu K: Structural comparison of three types of staphylococcal cassette chromosome mec integrated in the chromosome in methicillinresistant Staphylococcus aureus. Antimicrob Agents Chemother 2001 45(5):1323-1336

46. Diep BA, Stone GG, Basuino L, Graber CJ, Miller A, des Etages SA, Jones A, Palazzolo-Ballance AM, Perdreau-Remington F, Sensabaugh GF, et al: The arginine catabolic mobile element and staphylococcal chromosomal cassette mec linkage: convergence of virulence and resistance in the USA300 clone of methicillin-resistant Staphylococcus aureus. J Infect Dis 2008, 197(11):1523-1530.

47. Barragan MJ, Blazquez B, Zamarro MT, Mancheno JM, Garcia JL, Diaz E, Carmona M: BzdR, a repressor that controls the anaerobic catabolism of benzoate in Azoarcus sp. CIB, is the first member of a new subfamily of transcriptional regulators. J Biol Chem 2005, 280(11):10683-10694.

48. McCallum N, Hinds J, Ender M, Berger-Bachi B, Stutzmann Meier P: Transcriptional profiling of $\mathrm{XdrA}$, a new regulator of spa transcription in Staphylococcus aureus. J Bacteriol 2010, 192(19):5151-5164.

49. Ellison DW, Miller VL: Regulation of virulence by members of the MarR/ SlyA family. Curr Opin Microbiol 2006, 9(2):153-159.

50. Dorman CJ: Nucleoid-associated proteins and bacterial physiology. Adv Appl Microbiol 2009, 67:47-64.

51. Fuchs S, Pane-Farre J, Kohler C, Hecker M, Engelmann S: Anaerobic gene expression in Staphylococcus aureus. J Bacteriol 2007, 189(11):4275-4289.

52. Brown PH, Cronan JE, Grotli M, Beckett D: The biotin repressor: modulation of allostery by corepressor analogs. J Mol Biol 2004, 337(4):857-869.

53. Yang SJ, Rice KC, Brown RJ, Patton TG, Liou LE, Park YH, Bayles KW: A LysRtype regulator, $\mathrm{CidR}$, is required for induction of the Staphylococcus aureus cidABC operon. J Bacteriol 2005, 187(17):5893-5900.

54. Torrents E, Grinberg I, Gorovitz-Harris B, Lundstrom H, Borovok I, Aharonowitz Y, Sjoberg BM, Cohen G: NrdR controls differential expression of the Escherichia coli ribonucleotide reductase genes. J Bacteriol 2007, 189(14):5012-5021.

55. Schulz A, Schumann W: hrcA, the first gene of the Bacillus subtilis dnaK operon encodes a negative regulator of class I heat shock genes. J Bacteriol 1996, 178(4):1088-1093.

56. Liang X, Zheng L, Landwehr C, Lunsford D, Holmes D, Ji Y: Global regulation of gene expression by ArIRS, a two-component signal transduction regulatory system of Staphylococcus aureus. J Bacteriol 2005, 187(15):5486-5492.

57. Joshi GS, Spontak JS, Klapper DG, Richardson AR: Arginine catabolic mobile element encoded speG abrogates the unique hypersensitivity of Staphylococcus aureus to exogenous polyamines. Mol Microbiol 2011, 82(1):9-20.

58. Cue D, Lei MG, Luong $\Pi$, Kuechenmeister L, Dunman PM, O'Donnell S, Rowe S, O'Gara JP, Lee CY: Rbf promotes biofilm formation by Staphylococcus aureus via repression of icaR, a negative regulator of icaADBC. J Bacteriol 2009, 191(20):6363-6373.

59. Herron LL, Chakravarty R, Dwan C, Fitzgerald JR, Musser JM, Retzel E, Kapur V: Genome sequence survey identifies unique sequences and key virulence genes with unusual rates of amino Acid substitution in bovine Staphylococcus aureus. Infect Immun 2002, 70(7):3978-3981.

60. Ballal A, Manna AC: Expression of the sarA family of genes in different strains of Staphylococcus aureus. Microbiology 2009, 155(Pt 7):2342-2352.

61. Schmidt KA, Manna AC, Cheung AL: SarT influences sarS expression in Staphylococcus aureus. Infect Immun 2003, 71(9):5139-5148.

62. Schmidt KA, Manna AC, Gill S, Cheung AL: SarT, a repressor of alphahemolysin in Staphylococcus aureus. Infect Immun 2001, 69(8):4749-4758.

63. Kennedy AD, Bubeck Wardenburg J, Gardner DJ, Long D, Whitney AR, Braughton KR, Schneewind O, DeLeo FR: Targeting of alpha-hemolysin by active or passive immunization decreases severity of USA300 skin infection in a mouse model. J Infect Dis 2010, 202(7):1050-1058.

64. Liang $X$, Hall JW, Yang J, Yan M, Doll K, Bey R, Ji Y: Identification of single nucleotide polymorphisms associated with hyperproduction of alphatoxin in Staphylococcus aureus. PLoS One 2011, 6(4):e18428.

65. Fitzgerald JR, Monday SR, Foster TJ, Bohach GA, Hartigan PJ, Meaney WJ, Smyth CJ: Characterization of a putative pathogenicity island from bovine Staphylococcus aureus encoding multiple superantigens. J Bacteriol 2001, 183(1):63-70.
66. Finn RD, Tate J, Mistry J, Coggill PC, Sammut SJ, Hotz HR, Ceric G, Forslund K, Eddy SR, Sonnhammer EL, et al: The Pfam protein families database. Nucleic Acids Res 2008, 36(Database issue):D281-D288.

67. Perez-Rueda E, Collado-Vides J, Segovia L: Phylogenetic distribution of DNA-binding transcription factors in bacteria and archaea. Comput Biol Chem 2004, 28(5-6):341-350.

68. Moreno-Campuzano S, Janga SC, Perez-Rueda E: Identification and analysis of DNA-binding transcription factors in Bacillus subtilis and other Firmicutes-a genomic approach. BMC Genomics 2006, 7:147.

69. Kummerfeld SK, Teichmann SA: DBD: a transcription factor prediction database. Nucleic Acids Res 2006, 34(Database issue):D74-D81.

70. Janga SC, Moreno-Hagelsieb G: Conservation of adjacency as evidence of paralogous operons. Nucleic Acids Res 2004, 32(18):5392-5397.

doi:10.1186/1471-2164-14-126

Cite this article as: Ibarra et al:: Global analysis of transcriptional regulators in Staphylococcus aureus. BMC Genomics 2013 14:126.

\section{Submit your next manuscript to BioMed Central and take full advantage of:}

- Convenient online submission

- Thorough peer review

- No space constraints or color figure charges

- Immediate publication on acceptance

- Inclusion in PubMed, CAS, Scopus and Google Scholar

- Research which is freely available for redistribution 